\title{
Effects of Active Vibration Exercise on Trunk Muscle Activity, Balance, and Activities of Daily Living in Patients with Chronic Stroke
}

\author{
Dong-Kyu Lee', Eun-Kyung Kim² \\ 'Department of Physical Therapy, Sunhan Hospital, Gwangju; ${ }^{2}$ Department of Physical Therapy, Gangdong University, Chungbuk, Korea
}

Purpose: This study aimed to investigate the effect of active vibration exercise on trunk muscle activity, balance, and activities of daily living in patients with chronic stroke.

Methods: Twenty-four patients with chronic stroke were randomly assigned to an experimental $(n=12)$ or a control $(n=12)$ group. The experimental group performed an active vibration exercise workout for 20 minutes a day five times a week for 4 weeks using a flexi-bar. Trunk muscle activity was measured using surface electromyography. Balance was measured using the Berg balance scale. Activities of daily living were measured using the functional independence measure.

Results: The intragroup comparison showed significant differences in the rectus abdominis, internal oblique, and external oblique muscle activity values, Berg balance scale score, and functional independence measurement values in the experimental group. The intergroup comparison showed that differences in the rectus abdominis and internal oblique external oblique muscle values, Berg balance scale score, and functional independence measurement value for the experimental group were significantly related to those in the control group.

Conclusion: Based on these results, this study proved that the active vibration exercise effectively improved trunk muscle activity, balance, and activities of daily living in patients with chronic stroke.

Keywords: Active vibration exercise, Trunk muscle activity, Stroke

\section{INTRODUCTION}

Stroke is a disease caused by a loss of brain function induced by ischemia or cerebral hemorrhage of the blood vessels of the brain that leads to hemiplegia, negatively impacting trunk stability.,2 Stroke patients experience decreased function of the upper and lower limbs and muscle asymmetry, which weaken their trunk muscles and lead to a loss of trunk control function due to asymmetrical muscle activity., ${ }^{3,4}$ Abnormal muscle activities of the trunk impact one's proprioceptive sense by decreasing balance ability, hindering independent activities of daily living, and reducing quality of life. ${ }^{5,6}$

A safe and easy-to-use vibration exercise was recently applied to treat stroke patients. ${ }^{7}$ The vibration exercise improves the muscular strength and endurance by giving mechanical stimulus to sensory

Received Jul 26, 2018 Revised Aug 21, 2018

Accepted Aug 23, 2018

Corresponding author Eun-Kyung Kim

E-mail kek74ing@naver.com receptor such as muscle spindle through various amplitude and vibrations, thereby inducing reaction and adaptation of neuromuscular system. ${ }^{89}$ In contrast, the active vibration exercise allows the patients exercise in their own strength in various postures using a flexi-bar without external resistance. A flexi-bar is a $153 \mathrm{~cm}$ vibrating exercise device that uses $4.6 \mathrm{~Hz}$ vibrations generated by its shaking to help improve static and dynamic stability by controlling the vibration speed and amplitude generated actively by the subject rather than by manual vibration using a mechanical force. ${ }^{9}$ An active vibration exercise using flexi-bar can create coordination effect of muscles as the vibration generated while freely using the sagittal plane, transverse plane, and frontal plane make the agonistic and antagonistic muscles contract in turns to adjust instability during the exercise. ${ }^{910}$ Vibration exercise using a Flexi-Bar is reportedly ef-

Copylight (C2018 The Korea Society of Physical Therapy

This is an Open Access article distribute under the terms of the Creative Commons Attribution Non-commercial License (Http:// creativecommons.org/license/by-nc/4.o.) which permits unrestricted non-commercial use, distribution, and reproduction in any medium, provided the original work is properly cited. 
fective at improving trunk muscle thickness and balance. ${ }^{11}$ It has also been reported that exercise using a sling and flexi-bar is effective at reducing pain and increasing muscle activity in patients with rotator cuff repair. ${ }^{12}$ The flexi-bar is a vibration exercise tool that allows one to actively participate in the vibration exercise oneself without the need for expensive equipment or expert help., ${ }^{9,13}$ Most vibration exercises involve manual methods that directly cause vibration stimulation, whereas vibration exercises using the Flexi-Bar use active vibration stimulation.

However, few studies have examined the effect of active vibration exercises on chronic stroke patients' bodies. Therefore, this study aimed to analyze the effect of the active vibration exercise on chronic stroke patients' trunk muscle activities, balance, and activities of daily living.

\section{METHODS}

\section{Subjects}

The subjects of this study were 24 chronic stroke patients hospitalized in S hospital. This study divided 24 stroke patients into two groups: 12 (six men, six women) in the experimental group and 12 (six men, six women) in the control group. Subjects with no visual disability, with no orthopedic diseases in the upper or lower limbs, who scored more than 24 points on the mini-mental state examination, and who were able to walk more than $10 \mathrm{~m}$ using auxiliary equipment were included. All participants were informed of the study purpose and provided informed consent before the experiment. This study complied with the ethical standards of the declaration of Helsinki. For the experimental group the average age was $69.40 \pm 2.88$ years, the average height was $165.60 \pm 3.57 \mathrm{~cm}$, the average weight was $62.60 \pm 7.40 \mathrm{~kg}$, the average onset was $12.80 \pm 1.48$

Table 1. General characteristics of study subjects

$(n=24)$

\begin{tabular}{lccc}
\hline & EG $(n=12)$ & CG $(n=12)$ & $p$ \\
\hline Sex (male/female) & $6 / 6$ & $6 / 6$ & \\
Age (year) & $69.40 \pm 2.88$ & $68.20 \pm 1.64$ & 0.44 \\
Height (cm) & $165.60 \pm 3.57$ & $163.60 \pm 4.03$ & 0.43 \\
Weight (kg) & $62.60 \pm 7.40$ & $63.60 \pm 7.19$ & 0.83 \\
Onset (month) & $12.80 \pm 1.48$ & $13.40 \pm 1.34$ & 0.52 \\
MMSE-K (score) & $25.12 \pm 1.30$ & $24.20 \pm 1.64$ & 0.31 \\
\hline
\end{tabular}

Values are presented as mean \pm standard deviation.

EG: experimental group, CG: control group, MMSE-K: mini mental state examination-Korea. month and the average MMSE-K was $25.12 \pm 1.30$ score. For the control group the average age was $68.20 \pm 1.64$ years, the average height was $163.60 \pm 4.03 \mathrm{~cm}$, the average weight was $63.60 \pm 7.19 \mathrm{~kg}$, the average onset was $13.40 \pm 1.34$ month and the average MMSE-K was $24.20 \pm 1.64$ score (Table 1).

\section{Interventions}

The experimental and control groups received a neurodevelopmental treatment. In addition, the experimental group performed an active vibration exercise workout for 20 minutes a day five times a week for 4 weeks using a flexi-bar (FLEXI-BAR ${ }^{\circledR}$; Flexi-Sports, Germany), a stick that is $1,520 \mathrm{~mm}$ long and weighs $719 \mathrm{~g}$. The middle part of the stick has a rubber handle measuring $17.9 \mathrm{~cm}$, whereas the ends consist of weighty rubber, so the hands and arms transfer approximately $5 \mathrm{~Hz}$ vibrations when holding the middle handle and shaking the stick. The subject held the flexi-bar with both hands while standing and performed the up-and-down vibration exercise (Figure 1).

\section{Measurements}

Trunk muscle activity was measured using surface electromyography (LXM 5,308, Laxtha Inc., Korea). The configuration was set at a sampling rate of $1,024 \mathrm{~Hz}$, band pass filter of $20-450 \mathrm{~Hz}$, and notch filter of $60 \mathrm{~Hz}$. The obtained muscle activity signals were analyzed using the electromyography software (Telescan 3.11, Laxtha Inc., Korea) by root mean square processing. The body parts to which
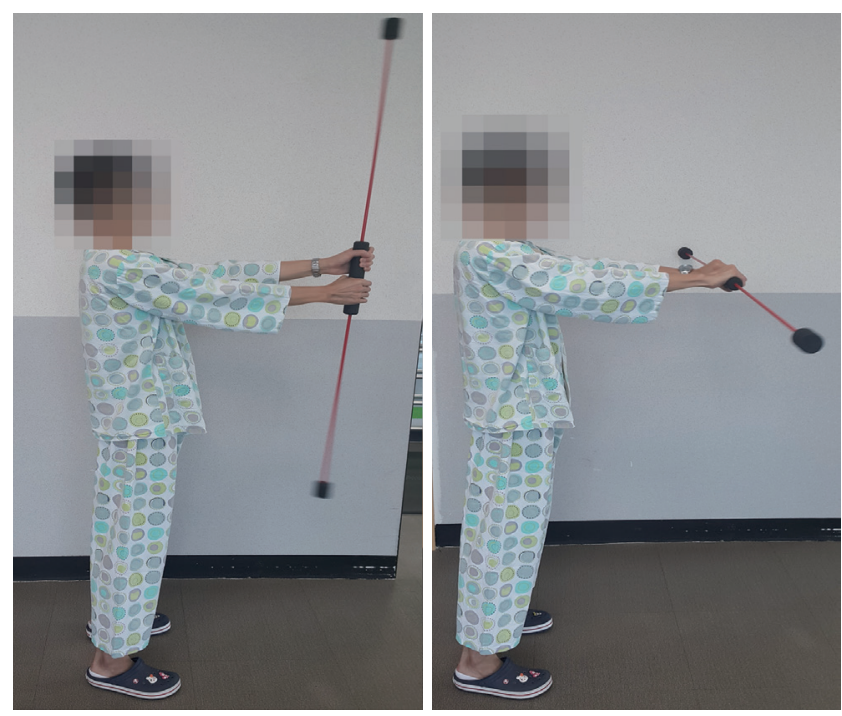

Figure 1. An active vibration exercise using flexi-bar. 
the surface electrodes were attached were rubbed with sandpaper and the horny layer of the skin was removed with cotton swabs containing alcohol to reduce the skin resistance. The surface electrodes were attached to the rectus abdominis (RA), external oblique (EO), and internal oblique (IO) muscles. To normalize the surface electromyography signal, a reference voluntary contraction was used. The reference contraction value was measured when the patient was comfortably seated on a chair, while the reference voluntary contraction value was measured when the patient stood up naturally from the seated position. The movements were measured thrice and the average value was used to minimize measurement errors, the measurement time was analyzed by collecting 3 -second signals that eliminated the first and last second and applying the percentage of the reference voluntary contraction value from the electromyography signal recorded by measuring for 5 seconds.

Balance was measured using the Berg balance scale (BBS), which included 14 items divided into three areas of sitting, standing, and posture change. The total possible score is 56 , the higher the score, the better the individual's balance.

Activities of daily living were measured using the functional independence measure (FIM), which consists of 13 items related to mobility and five related to recognition. The items were scored on a scale of 1-7 with 126 possible total points; higher scores indicate greater independence. The trunk muscle activity, BBS score and FIM score were recorded before and after the 4 weeks of treatment.

Table 2. The comparison of variable on pre and post in inner-group

\begin{tabular}{lccccc}
\hline & Group & Pre & Post & t & p \\
\hline RA (\%RVC) & EG & $39.50 \pm 2.25$ & $48.20 \pm 4.70$ & -9.41 & $0.01^{*}$ \\
& CG & $39.41 \pm 3.40$ & $41.24 \pm 2.83$ & -1.11 & 0.32 \\
IO (\%RVC) & EG & $26.04 \pm 3.46$ & $36.61 \pm 4.22$ & -4.49 & $0.01^{*}$ \\
& CG & $32.00 \pm 5.02$ & $33.75 \pm 2.85$ & -1.11 & 0.38 \\
EO (\%RVC) & EG & $35.79 \pm 3.66$ & $46.61 \pm 1.32$ & -5.72 & $0.05^{*}$ \\
& CG & $33.83 \pm 1.91$ & $37.88 \pm 2.71$ & -2.14 & 0.09 \\
BBS (score) & EG & $40.80 \pm 1.64$ & $44.80 \pm 0.83$ & -3.81 & $0.01^{*}$ \\
& CG & $39.20 \pm 2.48$ & $40.20 \pm 2.77$ & -2.23 & 0.08 \\
FIM (score) & EG & $82.40 \pm 2.30$ & $87.20 \pm 1.92$ & -3.07 & $0.03^{*}$ \\
& CG & $83.40 \pm 2.30$ & $83.80 \pm 1.92$ & -1.00 & 0.37
\end{tabular}

Values are presented as mean \pm standard deviation.

EG: experimental group, CG: control group, RA: rectus abdominis, IO: internal oblique, EO: external oblique, BBS: Berg balance scale, FIM: functional independence measure.

${ }^{*} p<0.05$.

\section{Statistical analysis}

SPSS software version 19.0 (SPSS Inc., Chicago, IL, USA) was used to statistically process and analyze the collected data. The Shapiro-Wilk test was used to check the normal distribution of the data. A paired ttest was performed to compare the extent of trunk muscle activity, balance, and activities of daily living in both groups before versus after the study. An independent t-test was used to compare intergroup differences, and the statistical significance level was set at $\alpha=0.05$.

\section{RESULTS}

\section{Trunk muscle activity}

\section{1) Rectus abdominis}

The experimental group had a significant difference as its rectus abdominis increased from $39.50 \pm 2.25$ to $48.20 \pm 4.70(\mathrm{p}<0.05)$, but the control group did not have a significant difference even though its rectus abdominis increased from $39.41 \pm 3.40$ to $41.24 \pm 2.83$ $(\mathrm{p}>0.05)$ (Table 2). The experimental group had more significant difference than the control group when the groups were compared before and after the experiment $(\mathrm{p}<0.05)$ (Table 3$)$.

\section{2) Internal oblique}

The experimental group had a significant difference as its internal oblique increased from $26.04 \pm 3.46$ to $36.61 \pm 4.22(\mathrm{p}<0.05)$, but the control group did not have a significant difference even though its internal oblique increased from $32.00 \pm 5.02$ to $33.75 \pm 2.85$ ( $\mathrm{p}>0.05)$ (Table 2). The experimental group had more significant difference than the control group when the groups were compared before and after the experiment $(\mathrm{p}<0.05)$ (Table 3$)$.

Table 3. The comparison of changes in variables between groups

\begin{tabular}{lcccc}
\hline & EG & CG & t & $p$ \\
\hline RA (\%RVC) & $12.70 \pm 3.01$ & $1.83 \pm 3.68$ & 5.10 & $0.00^{*}$ \\
IO (\%RVC) & $10.56 \pm 5.26$ & $1.74 \pm 3.51$ & 3.11 & $0.01^{*}$ \\
EO (\%RVC) & $10.82 \pm 4.22$ & $4.05 \pm 4.23$ & 2.52 & $0.03^{*}$ \\
BBS (score) & $4.00 \pm 2.34$ & $1.01 \pm 1.00$ & 2.63 & $0.03^{*}$ \\
FIM (score) & $4.80 \pm 3.49$ & $0.40 \pm 0.89$ & 2.72 & $0.02^{*}$ \\
\hline
\end{tabular}

Values are presented as mean \pm standard deviation.

EG: experimental group, CG: control group, RA: rectus abdominis, IO: internal oblique, EO: external oblique, BBS: Berg balance scale, FIM: functional independence measure.

${ }^{*} \mathrm{p}<0.05$. 


\section{3) External oblique}

The experimental group had a significant difference as its external oblique increased from $35.79 \pm 3.66$ to $46.61 \pm 1.32$ ( $\mathrm{p}<0.05)$, but the control group did not have a significant difference even though its external oblique increased from $33.83 \pm 1.91$ to $37.88 \pm 2.71(\mathrm{p}>0.05)$

(Table 2). The experimental group had more significant difference than the control group when the groups were compared before and after the experiment $(\mathrm{p}<0.05)$ (Table 3$)$.

\section{Balance}

The experimental group had a significant difference as its berg balance scale increased from $40.80 \pm 1.64$ to $44.80 \pm 0.83(\mathrm{p}<0.05)$, but the control group did not have a significant difference even though its berg balance scale increased from $39.20 \pm 2.48$ to $40.20 \pm 2.77$ $(\mathrm{p}>0.05)$ (Table 2). The experimental group had more significant difference than the control group when the groups were compared before and after the experiment $(\mathrm{p}<0.05)$ (Table 3).

\section{Activities of daily living}

The experimental group had a significant difference as it functional independence measure increased from $82.40 \pm 2.30$ to $87.20 \pm 1.92$ $(\mathrm{p}<0.05)$, but the control group did not have a significant difference although its functional independence measure decreased from 83.40 \pm 2.30 to $83.80 \pm 1.92(\mathrm{p}>0.05)$ (Table 2$)$. The experimental group had more significant difference than the control group when the groups were compared before and after the experiment $(\mathrm{p}<0.05)$ (Table 3$)$.

\section{DISCUSSION}

This study aimed to investigate the effect of active vibration exercise on trunk muscle activity, balance, and activities of daily living in patients with chronic stroke. Kim and Park ${ }^{14}$ conducted a study in a passive method using full-body vibration exercise equipment, while this study used a flexi-bar to apply active vibration exercise. In the intragroup comparison, there was a significant difference in the experimental group's trunk muscle activity, while in intergroup comparison, the experimental group's trunk muscle activities improved more significantly than those of the control group. When the flexi-bar exercise was performed by normal adults, a significant difference appeared in their trunk muscle activity. ${ }^{13}$ Moreside et al. ${ }^{10}$ reported that an active vibration exercise using a body blade had a positive effect on the improvements in trunk muscle activity, which was consistent with this study result. Moreover, the active vibration exercise using the flexi-bar effectively delivered the vibration stimulus to the trunk muscles, which enhanced proprioceptive sense and trunk muscle activity by intensively activating the muscle spindles. ${ }^{15}$ It also increased a-motor neuron mobilization and caused the tonic vibration reflex, a reflexive muscular contraction that then promoted muscle activity. ${ }^{16}$ Collectively, these results show that the active vibration exercise had a positive effect on improving trunk muscle activity.

$\mathrm{Kim}^{7}$ reported that stroke patients' balance ability improved after a vibration exercise. Another study using a flexi-bar to conduct vibration exercise in normal adults reported that their balance ability improved, supporting this study. ${ }^{11}$ Lee et al. ${ }^{11}$ reported that the balance ability improved when a flexi-bar was applied on normal people, while this study reported that the active vibration exercise using a flexi-bar improved the balance ability of chronic stroke patients. This result implies that active vibration exercise using flexi-bar is expected to be helpful for improving balance ability. In this study, the flexi-bar exercise, which results in active vibrations, gave a strong vibration stimulus to the trunk, contributed to trunk stability, and had a positive impact on nerve root adjustment, thereby enhancing balance.

Van Nes et al. ${ }^{17}$ analyzed in stroke patients' activities of daily living after a vibration exercise, thus supporting this study result. When the vibration stimulus was applied, the number of a-motor neurons increased, which positively impacted neuromuscular control, gross motor strength, and proprioceptive feedback. ${ }^{15,16}$ Bosco et al. ${ }^{16}$ suggested that vibration stimuli can increase muscular strength. In addition, active vibration stimuli can increase nerve control ability and muscular strength by causing co-contraction of a target muscle group. ${ }^{18}$ Based on these results, the active vibration exercise is thought to improve chronic stroke patients' physical functions and activities of daily living. This study confirmed that the active vibration exercise effectively enhances chronic stroke patients' trunk muscle activities, balance, and activities of daily living.

The limitation of this study is its short duration and lack of followup; hence, the long-term effects of the exercise protocol could not be determined. Moreover, the small sample size is insufficient to allow generalization of our results to all chronic stroke patients. Thus, an additional study to improve upon these problems is necessary. 


\section{REFERENCES}

1. Peurala SH, Könönen P, Pitkänen K et al. Postural instability in patients with chronic stroke. Restor Neurol Neurosci. 2007;25(2):101-8.

2. Jung KS, Kim YS, In TS. Effects of the support surface condition on muscle activity of trunk muscles during balance exercises in patients with stroke. J Kor Phys Ther. 2015;27(4):196-200.

3. Rathore SS, Hinn AR, Cooper LS et al. Characterization of incident stroke signs and symptoms: findings from the atherosclerosis risk in communities study. Stroke. 2002;33(11):2718-21.

4. Yang DJ, Uhm YH, Kim JH. The biofeedback scapular stabilization exercise in stroke patients effect of muscle activity and function of the upper extremity. J Kor Phys Ther. 2015;27(5):325-31.

5. Verheyden G, Vereeck L, Truijen S et al. Trunk performance after stroke and the relationship with balance, gait and functional ability. Clin Rehabil. 2006;20(5):451-8.

6. Lamb SE, Ferrucci L, Volapto S et al. Risk factors for falling in homedwelling older women with stroke: the women's health and aging study. Stroke. 2003;34(2):494-501.

7. Kim JH. The effects of whole body vibration exercise on balance and lower extremity muscle activity in stroke patients. J Kor Phys Ther. 2013; 25(5):266-72.

8. Torvinen S, Kannus P, Sievänen $\mathrm{H}$ et al. Effect of four-month vertical whole body vibration on performance and balance. Med Sci Sports Exerc. 2002;34(9):1523-8.

9. Mileva KN, Kadr M, Amin N et al. Acute effects of flexi-bar vs. shambar exercise on muscle electromyography activity and performance. J
Strength Cond Res. 2010;24(3):737-48.

10. Moreside JM, Vera-Garcia FJ, McGill SM. Trunk muscle activation patterns, lumbar compressive forces, and spine stability when using the bodyblade. Phys Ther. 2007;87(2):153-63.

11. Lee SJ, Kim YN, Lee DK. The effect of flexi-bar exercise with vibration on trunk muscle thickness and balance in university students in their twenties. J Phys Ther Sci. 2016;28(4):1298-302.

12. Kim JW, Kim YN, Lee DK. The effect of combined exercise with slings and a flexi-bar on muscle activity and pain in rotator cuff repair patients. J Phys Ther Sci. 2016;28(10):2890-3.

13. Um KM, Wang JS. Effect of flexi-bar exercise on postural alignment and balance ability in juvenile soccer players. JKAIS. 2015;16(8):5296-303.

14. Kim YM, Park JH. The effect of whole-body vibration exercise on balance, muscle strength and falls efficacy in the elderly. J Korean Soc Phys Med. 2017;12(4): 61-71.

15. Bogaerts A, Verschueren S, Delecluse C et al. Effects of whole body vibration training on postural control in older individuals: a 1 year randomized controlled trial. Gait Posture. 2007;26(2):309-16.

16. Bosco C, Colli R, Introini E et al. Adaptive responses of human skeletal muscle to vibration exposure. Clin Physiol. 1999;19(2):183-7.

17. van Nes IJ, Latour H, Schils F et al. Long-term effects of 6-week whole-body vibration on balance recovery and activities of daily living in the post-acute phase of stroke: A randomized, controlled trial. Stroke. 2006;37(9):2331-5.

18. Buteau JL, Eriksrud O, Hasson SM. Rehabilitation of a glenohumeral instability utilizing the body blade. Physiother Theory Pract. 2007;23(6):33349. 